\title{
Application of the Monte Carlo method in the calculation procedure of the internal combustion engine
}

The aim of the work is to check the possibility of applying the Monte Carlo method to the calculation procedure of internal combustion engines. This has been accomplished by modifying existing algorithms in such a way that the variables responsible for the main parameters of the engine are selected in a random manner using Solver written in Microsoft Excel. It turns out that this method can actually be implied, however, it has some limitations related to the high complexity of calculations.

Key words: internal combustion engine, Monte Carlo method, compression ignition engine, calculations

\section{Introduction}

The Monte Carlo method is one of the most popular methods of process modeling, mainly due to the ease of its application and the speed of obtaining results. Its areas of application include, among others, such areas as mass service systems, quality control and reliability, physics, chemistry, or even the real estate market. Among the above, mechanics and issues related to internal combustion engines are very rare, and it is a pity.

The Monte Carlo technique is a way to make a mathematical model of too complex processes, that is, the description of which would take too much time, or is simply impossible, due to the nature of the problem. The basis for this technique is the random selection of certain quantities that are responsible for the course of a given process. The accuracy of the results obtained depends on the number of attempts (but not always) and the quality of the pseudorandom number generator used, of course assuming that the draw takes place in accordance with a known distribution. Exploring the secrets of the mentioned method is very important in times of dynamic development, because it can enable us to "draw" a motor or another device with given parameters in just a few moments.

It would seem that we are slowly entering the era of electric vehicles, and internal combustion engines will lose their popularity in the coming years, but in reality there are a few issues with switching to fully electric means of transport. First of which is that a significant part of the world does not have infrastructure in the form of charging stations, and the amount of electricity produced is far from being needed to supply a huge amount of electric means of transport. Another difficulty is that the batteries weigh quite a lot these days and for example manufacturing a lorry that would have sufficient range would be neither possible nor profitable given the amount of batteries that would have to be used. All comes down to the energy equation, adding weight to any given vehicle makes it use more energy to move, and so far electric cars weigh undeniably more that their petrol powered counterparts. This leads to the issue of growing popularity of hybrid vehicles. In this case, the combustion engine usually works at a constant speed and load, and the energy it produces is used to charge the batteries, which power the electric motors, the main source of the drive. Such a scenario is perfect for the Monte Carlo method since it significantly simpli- fies calculations because the full range of rotational speeds and loads is not taken into account.

\section{Implementation of work}

\subsection{Calculation procedure}

The work is based largely on a computational program, written using Microsoft Excel, for compression ignition engines. The algorithms are relatively new, while the ranges of variable values have already been several or a dozen years old at the time of writing the program, so you can say that today they are not fully current data. For this reason, in the further part of the work, the impact of the change in the scope of these coefficients was checked.

Each of the sheets refers to a separate engine work phase, or related issues. The program consists of over 90 sheets, starting from pressures and temperatures in the processes of suction, compression, combustion and exhaust, going through the main geometric parameters, such as piston stroke and its diameter, all kinds of stresses and moments, to all the necessary details such as the dimensions of the crank head and foot or the entire geometry of the crankshaft. In this work, all attention is focused on the first few sheets of the program, and its goal is to generate an engine that consumes as much air as possible while taking the least fuel.

\subsection{Modification of the program}

To successfully prepare the program for the implementation of the Monte Carlo method, the first part of the program was imported to a single sheet, where it is easier to organize and modify all data. Then, after writing correctly functioning calculation algorithms, it was necessary to group and describe all variables, constants and results of calculations occurring in the tested part of the file. The result is a division into three groups (Tables 1-3).

\section{A) Variables}

This group includes all coefficients that will be randomly drawn by the generator, their values are chosen randomly, and the only condition is that they fit into particular ranges for each variable. In the initial phase of writing and modifying the program, there were fears that the compartments in which the above-mentioned coefficients lie are outdated, because they were determined several years ago. In order to check the above theory, each of the ranges of values was extended by about 10 to 25 percent, which un- 
fortunately did not affect the final result in any way. This means that the problem is more complex and you should check all possible configurations of changes, that is, change the ranges of one, several or each of the above coefficients, in all possible ways. Unfortunately, in a program such as Microsoft Excel, using only Solver would be too timeconsuming, and it is not known whether the results obtained would be satisfactory.

Table 1.Variables and ranges of their values

\begin{tabular}{|c|c|c|c|}
\hline \multicolumn{4}{|c|}{ Variables } \\
\hline Symbol & Name & Range & Unit \\
\hline$\eta_{v}$ & Fill factor & $0.7-0.9$ & \\
\hline $\mathcal{E}$ & Compression degree & $13-26$ & \\
\hline$\Delta T$ & $\begin{array}{l}\text { The temperature increase of the charge flowing into the } \\
\text { cylinder, caused by hot walls }\end{array}$ & $10-25$ & $\mathrm{~K}$ \\
\hline$p_{r}$ & Exhaust gas pressure & $0.105-0.12$ & $\mathrm{MPa}$ \\
\hline$T_{r}$ & The temperature of the residual fumes & $600-1000$ & $\mathrm{~K}$ \\
\hline$m_{s}$ & Exponent of polystyrene comp[ression & $1.27-1.38$ & \\
\hline$\overline{W_{u}}$ & The caloryfic value of fuel & $36-44$ & $\mathrm{MJ} / \mathrm{kg}$ \\
\hline$\xi$ & Coefficient of heat utilization & $0.7-0.9$ & \\
\hline$\chi_{s r}$ & Adiabatic exponent & $1.27-1.29$ & \\
\hline$m_{r}$ & Expansion exponent & $1.18-1.3$ & \\
\hline$V$ & theoretical volume of the stroke, suction and outlet loops & $0.92-0.97$ & \\
\hline$\eta_{m}$ & Mechanical efficiency of the engine & $0.7-0.9$ & \\
\hline
\end{tabular}

\section{B) Constants}

In this category, all constants taking part in the calculation process are included. The coefficient of excess air is in fact a variable value, however, it depends on the degree of pressure increase, so it can not be a random number, therefore some simplification was applied and the values closest to the average were selected.

Table 2. Constants and their values

\begin{tabular}{|c|l|c|c|}
\hline \multicolumn{3}{|c|}{ Constans } \\
\hline Symbol & Name & Value & Unit \\
\hline$p_{0}$ & Ambient pressure & 0.10 & $\mathrm{MPa}$ \\
\hline$T_{0}$ & Ambient temperature & & $\mathrm{K}$ \\
\hline$\lambda$ & Coefficient of excess air & 1.40 & \\
\hline$M$ & The theoretical ambient of air needed to burn of 1kg of fuel & 14.41 & $\mathrm{~kg}$ \\
\hline$\alpha$ & The degree of pressure increase & 1.90 & \\
\hline
\end{tabular}

C) Results

The last group contains all the values resulting from the random matching of variables in interaction with constants, this group defines to us whether the Monte Carlo method makes sense, because on the basis of these results we can determine if output values are achievable using today's technology.

Table 3. The results of subsequent actions on constants and variables

\begin{tabular}{|c|l|c|}
\hline \multicolumn{2}{|c|}{ Results } \\
\hline Symbol & Name & Unit \\
\hline$p_{1}$ & Pressure of the end of charging & $\mathrm{MPa}$ \\
\hline$T_{1}$ & The temperature of the end of charging & $\mathrm{K}$ \\
\hline$p_{2}$ & Pressure of the end of compression & $\mathrm{MPa}$ \\
\hline$T_{2}$ & The temperatuure of the end of compression & $\mathrm{K}$ \\
\hline$v_{0}$ & The specific volume of the ambient air & $\mathrm{m}^{3}$ \\
\hline$v_{k}$ & The volume of the compresssion chamber & $\mathrm{m}^{3}$ \\
\hline$P_{\text {max }}$ & Maximum of combustion pressure & $\mathrm{MPa}$ \\
\hline$\beta$ & Volume growth factor & \\
\hline$v_{4}$ & Gas volume at the theortical end of combustion & $\mathrm{m}^{3}$ \\
\hline$p_{5}$ & Pressure at the end of expansion & $\mathrm{MPa}$ \\
\hline$\varepsilon_{r}$ & Degree of expansion & \\
\hline$\dot{P}_{=}$ & Theoretical average of the induiicated pressure & $\mathrm{MPa}$ \\
\hline$\dot{p}_{i}$ & Average indicated pressure & $\mathrm{MPa}$ \\
\hline$p_{i}$ & Average usefull pressure & $\mathrm{MPa}$ \\
\hline Vs & The stroke volume nee ded to burn of 1 kg of fuel & $\mathbf{m}^{3}$ \\
\hline
\end{tabular}

\subsection{Solver}

Having already divided and grouped data, it is possible to move on to the most important element of the created program, namely the Solver add-in available in Excel. Thanks to this application, a subroutine has been written which will select random coefficients from the variable category based on a given result. In this case, the result is Vs, i.e. the stroke volume needed to burn $1 \mathrm{~kg}$ of fuel. This number should be as high as possible, because the goal is to use as much air and as little fuel as possible. In the Solver value (in this case it is Vs = maximum, marked in red, Fig. 1). Then all the restrictions are added (marked in yellow, Fig. 1), these are the ranges in which the variables contain. After determining the boundary conditions, the entire algorithm is being completed for all intermediate results, i.e. the remaining elements from the third group, because the final result depends on them, and they depend on the variables. The last step is to choose the solving method (marked in green, Fig. 1), Due to the high complexity of the problem, the evolutionary method was chosen.

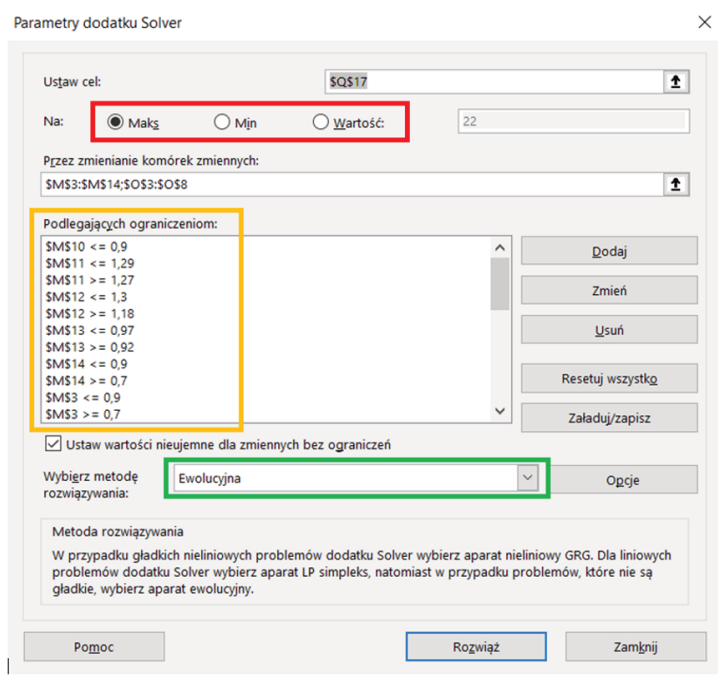

Fig. 1. View of the Solver add-in (print screen in polish)

\subsection{An example of implementing the Monte Carlo method}

After saving all algorithms and checking the operation of the program together with Solver, an exemplary application of the Monte Carlo method was developed. First step was finding the scope from the minimum to maximum value of Vs. Having found it, a certain value was chosen as the target, it was of course within the range from minimum to maximum, for example $0.023 \mathrm{~m}^{3}$. Further calculations were carried out, and Table 4. presents the coefficients obtained. Of course, finding a certain Vs is just one of the many possibilities of Solver, the operator of the program chooses which values will be drawn, and thus what the operation of the additive will be based on. The goal may be any element from the Results column, or even a different, more advanced indicator, however it will require extending the application with a further series of data. Another important factor is that the ranges in which coefficients are included are outdated, this means they should be updated before developing the program any further. Unfortunately addition, the final cell has been selected and given a certain 
this was not possible using Solver, because random changes in scopes would not provide valuable results, the issue of the ranges should be considered individually from scratch. However, the main purpose is to show the possibilities of applying the Monte Carlo method, accuracy of the results is in this case a secondary matter. The more expanded the program, the larger the area of application can be covered, it is possible to write a program that will "draw" the entire engine, including the geometry.

Table 4. Values of the variables and results for Vs $=0.023$
\begin{tabular}{|c|r|c|r|c|r|}
\hline \multicolumn{2}{|c|}{ Variables } & \multicolumn{2}{c|}{ Constants } & \multicolumn{2}{c|}{ Results } \\
\hline$\eta_{v}$ & 0.70 & $p_{0}$ & 0.10 & $p_{1}$ & 0.08 \\
\hline$\varepsilon$ & 18.29 & $T_{0}$ & 298.00 & $T_{1}$ & 325.58 \\
\hline \hline$\Delta$ & 10.55 & $\lambda$ & 1.40 & $p_{2}$ & 4.11 \\
\hline$p_{r}$ & 0.12 & $M_{t}$ & 14.41 & $T_{2}$ & 974.20 \\
\hline$T_{r}$ & 768.75 & $\alpha$ & 1.90 & $v_{0}$ & 0.86 \\
\hline$m_{s}$ & 1.38 & $l$ & 1.00 & $V_{k}$ & 1.43 \\
\hline$W_{u}$ & 38.90 & & & $p_{\max }$ & 7.81 \\
\hline$\xi$ & 0.77 & & & $\beta$ & 1.23 \\
\hline \hline$\chi_{s r}$ & 1.28 & & & $v_{4}$ & 1.75 \\
\hline$m_{r}$ & 1.19 & & & $p_{5}$ & 0.31 \\
\hline$V$ & 0.93 & & & $\varepsilon_{r}$ & 14.93 \\
\hline$\eta_{m}$ & 0.77 & & & $\dot{P}_{2}$ & 0.85 \\
\hline & & & & $\dot{p}_{i}$ & 0.75 \\
\hline & & & & $p_{i}$ & 0.58 \\
\hline & & & & $V_{s}$ & 24.65 \\
\hline
\end{tabular}

Having a certain value of Vs and all other coefficients, last thing left to do to get the outline of the indicator graph is to determine the basic parameters defining the engine, such as engine power, number of cylinders, rotational speed or fuel consumption. Of course, also these values can be freely modified, depending on the needs. After setting these parameters, the result of the calculation was generated in the form of the main points of the indicator chart, referring to the maximum and minimum pressures, volumes and temperatures in the engine's work cycle. Based on them, it is possible to predict the course of these parameters and draw a graph showing the way this engine works.

Table 5. Results of the calculation, main points of the indicator chart

\begin{tabular}{|c|r|r|r|r|r|r|}
\hline Variable & \multicolumn{1}{c|}{$\mathrm{cm}^{3}$} & Variable & \multicolumn{1}{|c|}{ Mpa } & Variable & \multicolumn{1}{c|}{$\mathrm{K}$} & ${ }^{\circ} \mathrm{C}$ \\
\hline $\mathrm{V} 1=$ & 830.97 & $\mathrm{P} 1=$ & 0.08 & $\mathrm{~T} 1=$ & 322.01 & 48.86 \\
\hline $\mathrm{V} 2=$ & 32.50 & $\mathrm{P} 2=$ & 5.07 & $\mathrm{~T} 2=$ & 801.51 & 528.36 \\
\hline $\mathrm{V} 3=$ & 32.50 & $\mathrm{P} 3=$ & 9.64 & $\mathrm{~T} 3=$ & 1521.75 & 1248.60 \\
\hline $\mathrm{V} 4=$ & 46.02 & $\mathrm{P} 4=$ & 9.64 & $\mathrm{~T} 4=$ & 2154.78 & 1881.63 \\
\hline $\mathrm{V} 5=$ & 830.97 & $\mathrm{P} 5=$ & 0.25 & $\mathrm{~T} 5=$ & 947.12 & 673.97 \\
\hline $\mathrm{V} 6=$ & 830.97 & $\mathrm{P} 6=$ & 0.11 & & & \\
\hline $\mathrm{V} 7=$ & 32.50 & $\mathrm{P} 7=$ & 0.11 & & & \\
\hline
\end{tabular}

The above illustration shows the 7 most important values of volume and pressures and 5 temperatures. For volume, they are the maximum cylinder volume at the end of charging and expansion $\left(\mathrm{V}_{1}, \mathrm{~V}_{6}, \mathrm{~V}_{7}\right)$, then at the end of compression $\left(\mathrm{V}_{2}, \mathrm{~V}_{3}, \mathrm{~V}_{7}\right)$, and the volume at the theoretical end of combustion $\left(\mathrm{V}_{4}\right)$. On the other hand, the pressures are distinguished as the end of charging pressure ( $\left.\mathrm{p}_{1}\right)$, during compression $\left(\mathrm{p}_{2}\right)$, the maximum combustion pressure (p3, p4), expansion (p5) and exhaust gas pressure (p6, p7).

Temperatures in turn result from increases in volume as well as pressures and the shape of the compression and expansion curves. The first one is the temperature at the end of charging ( $\left.\mathrm{T}_{1}\right)$, then the compression ( $\left.\mathrm{T}_{2}\right)$. $\mathrm{T}_{3}$ is the result of multiplying the previous one $\left(\mathrm{T}_{2}\right)$ by the degree of pressure increase $(\alpha), T_{4}$ in turn is equal to $T_{3}$ times the volume increase factor. The last of the temperatures (T5) also includes the exponent of the expansion, which determines how much a drop in temperature after the combustion process is achieved.

\section{Conclusions}

The main purpose of the work was to check the possibility of applying the Monte Carlo method to the calculation of internal combustion engines, in this case the self-ignition engine. From the above, it can be seen that this method is possible and, moreover, relatively easy to apply.

Although the results obtained are not sufficient to design the entire engine, including geometry, they help determine what should be guided in the design and construction process. It was also found that the Monte Carlo technique has an incredibly wide range of applications and should be consistently implemented in more and more fields of science. A huge plus of this method, despite the low accuracy of results, is that in a very short time we get some results that shape the view of the rest of the problem. It is much easier to carry out further research knowing what it is aiming for and having already this initial "piece of success".

Returning to the engines, if the decision was made to design the motor based on the known method, the focus should be on maximizing the program, taking into account forces and stresses, as well as the entire geometry. Of course, not all variables can be simply set on the level drawn, most of them depend on many other factors that should be known first. Only when we know how to influence such values as exhaust gas pressure or the exponent of pressure polystyrene and many others, we will find out whether it is worth striving for those determined on the basis of a draw, or perhaps it would be better to sometimes use a classic calculation model. After finding the golden mean, between the Monte Carlo method and the analytical approach, we get a calculation program for internal combustion engines, combining satisfactory speed of calculation and sufficient accuracy of results.

\section{Bibliography}

[1] JANICKA, A., KOLANEK, C., WALKOWIAK, W. Applied Thermodynamics - internal combustion engine Laboratory.

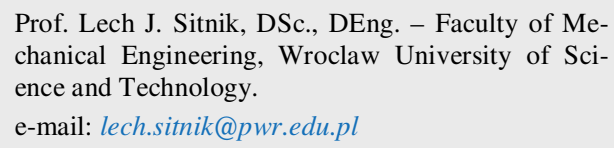

[2] Owen Art. Monte Carlo Methods. 2002.

[3] SITNIK L. Program obliczeniowy silnika spalinowego o zapłonie samoczynnym, Wrocław 2007.
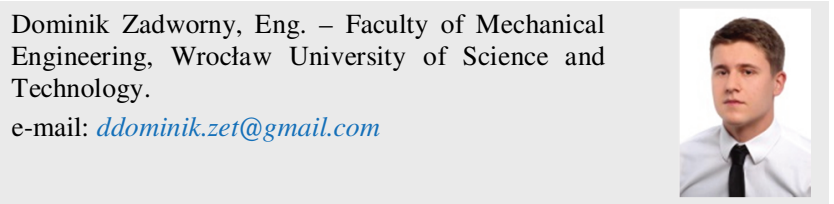